\title{
AN EFFECTIVE OPERATOR, CONTINUOUS BUT NOT PARTIAL RECURSIVE
}

\author{
PAUL R. YOUNG ${ }^{1}$
}

Introduction. It is known that under many conditions, effective operators will be partial recursive, ([MS], [KLS], [L]). On the other hand, certain pathological examples have been constructed by Friedberg $[\mathbf{F}]$ and Pour-El $[\mathrm{P}]$ to show that effective operators are not always partial recursive. Pour-El has observed that although it is well known that all partial recursive operators are continuous, the effective but not partial recursive operators of $[\mathbf{F}]$ and $[\mathbf{P}]$ are not continuous, and she has raised the question of the existence of effective operators which are continuous but not partial recursive. It is easy to see that all partial recursive operators are not just continuous, but are in fact "effectively continuous." This enables us to answer Pour-El's question by constructing an effective operator which is continuous but not "effectively continuous." Since it is continuous, our example of an effective but not partial recursive operator is perhaps less pathological than earlier examples. ${ }^{2}$

Notation and definitions. $N$ is the set of all nonnegative integers. $\odot$ is the set of all partial functions mapping $N$ to $N$, and $\mathcal{P r}^{r}$ is the set of all partial recursive elements of $\mathcal{P}$. $\left\{\phi_{e}\right\}$ is a standard effective enumeration of $\rho^{r}$. In this paper we will be concerned only with operators mapping subsets of $\mathcal{P}^{r}$ into $\mathcal{P}^{r}$.

We will let $f$ be a fixed total recursive function for which $\left\{\phi_{f(i)}\right\}$ is a one-one enumeration of all finite members of $\rho$ (equivalently of $\left.\odot^{r}\right)$ and for which each $f(i)$ is a canonical index; i.e., we can effectively compute the cardinality of $\phi_{f(i)}$ from $f(i)$.

$\beta$ is topologized by taking as basic open sets all sets of the form $\left\{\psi \mid \phi_{f(i)} \subseteq \psi\right\}$ and we denote $\left\{\psi \mid \phi_{f(i)} \subseteq \psi\right\}$ by $\phi_{f(i)}$. This topology yields a relative topology on $\mathcal{P}^{r}$ and we denote $\phi_{f(i)} \cap \rho^{r}=\left\{\phi_{e} \mid \phi_{f(i)} \subseteq \phi_{e}\right\}$ also by $\phi_{f(i)}$, relying on the context to make the usage clear.

Intuitively, we want an operator $\Phi$ with domain $D \subseteq \mathcal{P}$ to be effectively continuous if, given $\psi \in D$ and a neighborhood $M$ of $\Phi(\psi)$, we

Received by the editors October 13, 1966.

1 Research and publication supported by a National Science Foundation Postdoctoral Fellowship at Stanford University and by NSF Research Grant 2754.

${ }^{2}$ The referee informs me that C. E. M. Yates has also constructed an example (unpublished) of an effective operator which is continuous but not partial recursive. 
can effectively find a neighborhood $M^{\prime}$ of $\psi$ such that $\psi^{\prime} \in M^{\prime} \cap D$ implies that $\Phi\left(\psi^{\prime}\right) \in M$. However we restrict ourselves to working with domains in $\rho^{r}$ and to basic open sets as neighborhoods. For the purpose of this paper, we adopt the following

Definition. An operator $\Phi$ on the domain $D \subseteq \mathcal{P}^{r}$ is effectively contimuous if there is a partial recursive function $c(e, y)$ such that, if $\phi_{e} \in D$ and $\Phi\left(\phi_{e}\right) \in \phi_{f(y)}$, then $c(e, y)$ is defined, $\phi_{e} \in \phi_{f(c(e, y))}$, and if $\phi_{x} \in \phi_{f(c(e, y))} \cap D$ then $\Phi\left(\phi_{x}\right) \in \phi_{f(y)}$.

We remark in passing that an effectively continuous operator need not be an effective operator, even when its range is contained in $\mathbb{P}^{r}$. To see this, we consider the domain $D$ consisting of all finite functions and consider operators mapping $D$ into $D$. For each set $S \subseteq N$ we define the operator $\Phi_{S}$ by

$$
\Phi_{S}\left(\phi_{e}\right)=\phi_{e} / S
$$

where $\phi_{e} / S$ denotes the restriction of $\phi_{e}$ to $S$. One easily verifies that distinct sets $S$ and $S^{\prime}$ give rise to distinct operators $\Phi_{S}$ and $\Phi_{S^{\prime}}$. Furthermore each such operator is effectively continuous because the identity function $c(e, y)=y$ witnesses the effective continuity of each $\Phi_{S}$. Since there are uncountably many operators $\Phi_{S}$ but only countably many effective operators on any given domain, not all effectively continuous operators can be effective operators. On the other hand, it may be that placing additional conditions on the operator will assure that effective continuity of an operator will imply effectiveness of the operator. For example, we do not know whether every effectively continuous operator mapping all of $\rho^{r}$ into $\rho^{r}$ is effective. We also lack an example of an effectively continuous effective operator which is not partial recursive.

Definition, [MS]. An operator $\Phi$ is effective on the domain $D \subseteq \mathcal{P}^{r}$ if there is a total recursive function $g$ such that for all $\phi_{e} \in \mathcal{D}$,

$$
\Phi\left(\phi_{e}\right)=\phi_{g(e)} .
$$

REMARK. An immediate consequence of this definition is that if $\phi_{e} \in \mathbb{D}$ and $\phi_{x}=\phi_{e}$ then $\phi_{g(x)}=\phi_{g(e)}$.

Lemma 1. Let $\Phi$ be a partial recursive operator on the domain D. If $D^{\prime}=\left\{\phi_{x} \mid \phi_{x} \subseteq \phi_{e}\right.$ for some $\left.\phi_{e} \in \mathbb{D}\right\}, \Phi$ can be extended to a partial recursive operator on $D^{\prime}$ and there is a total recursive function $g$ such that, for all $\phi_{e} \in \mathfrak{D}^{\prime}, \Phi\left(\phi_{e}\right)=\phi_{g(e)}$.

Finally, if $t$ is a recursive function such that

$$
\phi_{t(n)} \subseteq \phi_{e} \in D^{\prime}, \quad \text { and } \lim _{n} \phi_{t(n)}=\phi_{e},
$$

then 


$$
\phi_{\theta(t(n))} \subseteq \phi_{g(e)}, \quad \text { and } \lim _{n} \phi_{g(t(n))}=\phi_{g(e)} .
$$

Proof. This is well known. E.g., it is an immediate consequence of the definition of partial recursive operator and Lemmas 3.1' and 3.2' given in [L], (bearing in mind that every partial recursive operator is a Banach-Mazur operator). Since we will be working only with domains and ranges contained in $\rho^{r}$, the reader unfamiliar with partial recursive operators may take the existence of such extensions as the defining property for partial recursive operators.

LEMMA 2. Every partial recursive operator is effectively continuous on its domain.

Proof. We simply give the usual proof of continuity, observing that the calculations are effective: Let $\Phi$ be partial recursive on the

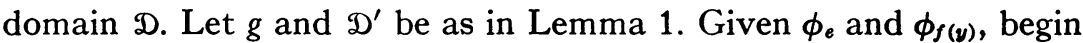
enumerating $\phi_{e}$, letting

$$
\phi_{f(h(n, 0))}=\phi_{e}^{(n)}=\text { the set of elements of } \phi_{e} \text { enumerated in } \phi_{e} \text { by stage } n \text {. }
$$

By Lemma 1,

$$
\phi_{g(e)}=\lim _{n} \phi_{g(f(h(n, e)))}
$$

if $\phi_{e} \in \mathfrak{D}^{\prime}$, so if $\phi_{f(y)} \subseteq \phi_{g(e)}$ we eventually find $n_{0}$ such that $\phi_{f(y)}$ $\subseteq \phi_{g\left(f\left(h\left(n_{0}, e\right)\right)\right)}$. Also by Lemma 1, if $\phi_{f\left(h\left(n_{0}, e\right)\right)} \subseteq \phi_{z}$ and $\phi_{2} \in D^{\prime}$ then $\phi_{g\left(h\left(n_{0}, e\right)\right)} \subseteq \phi_{g(z)}$. Thus if we define $\phi_{c(e, y)}=\phi_{f\left(h\left(n_{0}, e\right)\right)}, c$ will witness the effective continuity of $\Phi$.

The proof of our result blends two techniques. One is the technique introduced by Friedberg to construct effective operators which are not partial recursive. The other is a rate-of-growth argument: Given a basic open set $\phi_{f(y)}$ in the range of the operator $\Phi$ we are going to find a basic open set $\phi_{f(c(e, y))}$ in the domain of $\Phi$ in such a way that the function $c$ will establish the continuity of $\Phi$. However, to establish continuity, for some fixed $e$ the rate of growth of $\left|\phi_{(f(e, y))}\right|$ with respect to $\left|\phi_{f(y)}\right|$ will have to be so great that $c$ cannot be a recursive function. $\left(\left|\phi_{f(x)}\right|\right.$ is the cardinality of $\phi_{f(x) .}$.)

TheOREM. (A) There is an effective operator, $\Phi$, which is continuous on its domain but which is not effectively continuous. ( $A$ fortiori, $\Phi$ is not partial recursive.)

((B). The operator $\Phi$ of $(\mathrm{A})$ has the following property: It is the union of a partial recursive operator $\Phi_{0}$ on a completely recursively enumerable domain, $\mathcal{e}$, together with the trivial operator $\Phi_{1}(\omega)=\omega$ defined only on a certain constant function $\omega$.) 
(REMARK. The relevance of $(B)$, whose proof the reader may ignore in proving (A), is that any effective operator $\Phi_{0}^{\prime}$ on a completely recursively enumerable domain $\mathcal{C}$ may be trivially extended to an effective operator $\Phi_{0}^{\prime}$ on all of $\rho^{r}$ simply by defining $\Phi_{0}^{\prime}\left(\phi_{x}\right)$ to be the nowhere defined function for $\phi_{x} \in \mathcal{C}$. By [MS], any effective operator defined on all of $\rho^{r}$ is in fact partial recursive, and hence effectively continuous. Thus $\Phi$, which differs almost trivially from $\Phi_{0}$, is not effectively continuous even though $\Phi$ is continuous and $\Phi_{0}$ can be extended to an effectively continuous operator on all of $\boldsymbol{P r}^{\mathrm{r}}$.)

Proof. Let $\psi$ be a partial recursive function which can be majorized by no total recursive function. (E.g., if $\psi(x)=\phi_{x}(x)+1$, the assumption that $\phi_{e}$ is a total function majorizing $\psi$ leads to an immediate contradiction.)

An element $m$ of the domain of $\psi$ is called maximal if $n<m$ implies $\psi(n)<\psi(m)$ wherever $\psi(n)$ is defined. It is easy to see that any function with a largest maximal element is bounded. Therefore $\psi$ has no largest maximal element. Since $n=(\mu y)[\psi(y)$ is defined] is maximal, $\psi$ has infinitely many maximal elements.

We cannot define the operator $\Phi$ which we are seeking directly from the enumerations of the $\phi_{e}$ 's, for if we $\operatorname{did} \Phi$ would be partial recursive. Consequently we adopt the technique introduced by Friedberg in [F] to produce effective operators which are not partial recursive. We let $R=\left\{e \mid \phi_{e}(x)=0\right.$ for all $\left.x \leqq e\right\}$ and let $\omega$ be the function $\omega(x)=0$ for all $x$. We let $\omega_{n}$ be the function $\{\langle 0,0\rangle,\langle 1,0\rangle, \cdots,\langle n, 0\rangle\}$ $=\omega /\{0,1, \cdots, n\}$.

We now construct the total recursive function $g$ which computes $\Phi$.

We find it convenient to use a marker, $\Lambda$, in the course of construction. Although distinct members may be simultaneously marked by $\Lambda$, once $\Lambda$ is introduced beside a number it is never moved from the number nor are priority methods used in the construction.

First begin enumerating $R$; whenever we find $e \in R$ we place $\omega$ into $\phi_{g(e)}$.

We also enumerate $\psi$ and whenever we find $m$ in the domain of $\psi$ we look for the smallest $r>0$ such that

$$
\phi_{f(i)}={ }_{\operatorname{def}} \omega_{\psi(m)-1} \cup\{\langle\psi(m), r\rangle\}
$$

is not yet known to have an extension $\phi_{e}$ with $e \in R$ and such that the marker $\Lambda$ does not appear beside the canonical index $f(i)$. We then place the marker $\Lambda$ beside $f(i)$ and we place $\omega_{m-1}$ into $\phi_{g(a)}$ for every extension $\phi_{a}$ of $\phi_{f(i)}$.

For each $f(i)$ with the marker $\Lambda$ beside it, $\phi_{f(i)}$ defined by $\left({ }^{*}\right)$, we also do the following: If we find $e \in R$ with $\phi_{e}$ an extension of $\phi_{f(i)}$, 
we place $\omega$ into $\phi_{g(a)}$ for every extension $\phi_{a}$ of $\phi_{f(i)}$. When this occurs, we also find the smallest $r^{\prime}>0$ for which

$$
\phi_{f\left(i^{\prime}\right)}=\operatorname{def} \omega_{\psi(m)-1} \cup\left\{\left\langle\psi(m), r^{\prime}\right\rangle\right\}
$$

is not yet known to have an extension $\phi_{e}$ with $e \in R$ and such that the marker $\Lambda$ has not been placed beside the canonical index $f\left(i^{\prime}\right)$. We place the maker $\Lambda$ beside $f\left(i^{\prime}\right)$ and we place $\omega_{m-1}$ into $\phi_{g(a)}$ for every extension $\phi_{a}$ of $\phi_{f\left(i^{\prime}\right)}$.

This completes our description of $g$.

Let $\mathcal{C}=\left\{\phi_{e} \mid \phi_{e}\right.$ extends some $\phi_{f(i)}$ where $f(i)$ has the marker, $\Lambda$, placed beside it in the course of the construction\}. (Since $\mathcal{C}$ is the class of all r.e. supersets of a r.e. sequence of canonically enumerable finite sets, $\mathfrak{C}$ is completely recursively enumerable by a standard characterization of completely enumerable classes given in [MS] and $[R]$.)

It is clear from our construction of $g$ that if $\phi_{x}=\phi_{y}$ and if $\phi_{x} \in \mathfrak{C}$, then $\phi_{g(x)}=\phi_{g(y)}$. (In fact we know that either $\phi_{g(x)}=\omega$ or $\phi_{g(x)}=\omega_{m-1}$ for some $m$ in the domain of $\psi$.) Also, if $\phi_{e}=\omega$, then $e \in R$, so that $\phi_{g(e)}=\omega$. Thus $g$ determines an effective operator, $\Phi$, on $\mathcal{e} \cup\{\omega\}$.

We now show that $\Phi$ is continuous at each point of its domain, $\mathcal{e} \cup\{\omega\}$. If $\phi_{x} \in \mathcal{C}$, then for some $z$ belonging to the domain of $\psi$ and for some $r>0, \phi_{x}$ extends $\omega_{\psi(z)-1} \cup\{(\psi(z), r)\}$. Furthermore, for all $y$ such that $\phi_{y}$ extends $\omega_{\psi(z)-1} \cup\{(\psi(z), r)\}, \phi_{\nu} \in \mathbb{C}$ and

$$
\Phi\left(\phi_{y}\right)=\Phi\left(\omega_{\psi(z)-1} \cup\{(\psi(z), r)\}\right) .
$$

Thus if $\phi_{f(i)} \subseteq \Phi\left(\phi_{x}\right)$ and $\omega_{\psi(z)-1} \cup\{(\psi(z), r)\} \subseteq \phi_{y}$, then $\phi_{f(i)} \subseteq \Phi\left(\phi_{y}\right)$. This establishes that $\Phi$ is continuous at each point of $\mathcal{C}$. To establish continuity at $\omega$, suppose $\phi_{f(i)} \subseteq \Phi(\omega)(=\omega)$. Let $n_{i}$ be a maximal element of the domain of $\psi$ such that $\phi_{f(i)} \subseteq \omega_{n_{i}-1}$. Clearly $\omega_{\psi\left(n_{i}\right)-1} \subseteq \omega$. Suppose $\phi_{x} \in \mathcal{C}$ and $\omega_{\psi\left(n_{i}\right)-1} \subseteq \phi_{x}$. Then there is an element $z$ belonging to the domain of $\psi$ and an $r>0$ such that $\omega_{\psi(z)-1} \cup\{(\psi(z), r)\} \subseteq \phi_{x}$. By the construction,

$$
\omega_{z-1} \subseteq \Phi\left(\omega_{\psi(z)-1} \cup\{(\psi(z), r)\}\right)=\Phi\left(\phi_{x}\right) .
$$

Since $\psi\left(n_{i}\right) \leqq \psi(z)$ and $n_{i}$ is maximal, $n_{i} \leqq z$. Thus

$$
\phi_{f(i)} \subseteq \omega_{n_{i}-1} \subseteq \omega_{z-1} \subseteq \Phi\left(\phi_{x}\right),
$$

establishing the continuity of $\Phi$ at $\omega$.

It remains to show that $\Phi$ is not effectively continuous on $\mathcal{e} \cup\{\omega\}$.

We first show that for each $m$ in the domain of $\psi$ there is some extension of $\omega_{\psi(m)-1}$ which gets mapped to $\omega_{m-1}$. It is in fact clear from our construction that this will happen unless $\omega_{\psi(m)-1} \cup\{\langle\psi(m), r\rangle\}$ 
gets mapped to $\omega$ for infinitely many $r>0$. But for $\omega_{\psi(m)-1} \cup\{\langle\psi(m), r\rangle\}$ to get mapped to $\omega(r>0)$, there must be some $\phi_{e}$ extending $\omega_{\psi(m)-1}$ $\cup\{\langle\psi(m), r\rangle\}$ with $e \in R$, i.e. with $\phi_{e}(x)=0$ for all $x \leqq e$. Since this implies that $e<\psi(m)$, there are at most finitely many such $e$ 's, and so for each $m$ in the domain of $\psi$, there is some $r>0$ with $\omega_{\psi(m)-1}$ $\cup\{\langle\psi(m), r\rangle\}$ mapped to $\omega_{m-1}$.

Now suppose that $\Phi$ were effectively continuous. Since $\Phi(\omega)=\omega$, given $n$, since $\omega_{n} \subseteq \omega$, we could effectively find $\phi_{f(s(n))} \subseteq \omega$ such that each extension of $\phi_{f(s(n))}$ in the domain of $\Phi$ has an image which extends $\omega_{n}$. Letting $t$ be the total recursive function such that

$$
t(n)=\max \left\{y \mid\langle y, 0\rangle \in \phi_{f(s(n))}\right\}
$$

we would have that $\omega_{t(n)} \subseteq \phi_{e}$ and $\phi_{e} \in \mathcal{C} \cup\{\omega\}$ implies $\omega_{n} \subseteq \Phi\left(\phi_{e}\right)$. But for $m$ in the domain of $\psi$ there is some $r>0$ such that

$$
\Phi\left(\omega_{\psi(m)-1} \cup\{\langle\psi(m), r\rangle\}\right)=\omega_{m-1} .
$$

Since $t(m)<\psi(m)$ implies

$$
\omega_{t(m)} \subseteq \omega_{\psi(m)-1} \cup\{\langle\psi(m), r\rangle\},
$$

$t(m)<\psi(m)$ implies $\omega_{m} \subseteq \omega_{m-1}$, a contradiction. Thus $t(m) \geqq \psi(m)$ for all $m$ for which $\psi(m)$ is defined. This means that $t$ majorizes $\psi$, and this contradiction shows that $\Phi$ is not effectively continuous, completing our proof.

\section{REFERENCES}

[F] R. S. Friedberg, Un contre-example relatif aux fonctionelles recursives, C. R. Acad. Sci. Paris 247 (1958), 852-854.

[KLS] G. Kreisel, D. Lacombe and J. R. Shoenfield, "Partial recursive functionals and effective operations" in Constructivity in mathematics, North-Holland, Amsterdam, 1959, pp. 290-297.

[L] A. N. Lachlan, Effective operations in a general setting, J. Symbolic Logic 29 (1964), 163-178.

[MS] J. Myhill and J. C. Shepherdson, Effective operations on partial recursive functions, Z. Math. Logik Grundlagen Math. 1 (1955), 310-317.

[P] M. B. Pour-El, A comparison of five computable operators, Z. Math. Logik Grundlagen Math. 6 (1960), 325-340.

[R] H. G. Rice, Completely recursively enumerable classes and their key arrays, J. Symbolic Logic 74 (1953), 358-366.

Purdue University 